\title{
FLYING CASSINI WITH VIRTUAL OPERATIONS TEAMS
}

\author{
Suzanne Dodd* and Robert Gustavson** \\ *Jet Propulsion Laboratory \\ California Institute of Technology \\ National Aeronautics and Space Administration \\ 4800 Oak Grove Drive M/S 230-101, Pasadena, CA 91109-8099 USA \\ suzanne.r.dodd@jpl.nasa.gov, +1 (818) 393-5865, Fax +1 (818) 393-1105 \\ **Jet Propulsion Laboratory \\ California Institute of Technology \\ National Aeronautics and Space Administration \\ 4800 Oak Grove Drive M/S 230-101, Pasadena, CA 91109-8099 USA \\ robert.p.gustavson@jpl.nasa.gov, +1 (818) 393-3289,Fax +1 (818) 393-1105
}

\begin{abstract}
The Cassini Program's challenge is to fly a large, complex mission with a reduced operations budget. A consequence of the reduced budget is elimination of the large, centrally located group traditionally used for uplink operations. Instead, responsibility for completing parts of the uplink function is distributed throughout the Program. A critical strategy employed to handle this challenge is the use of Virtual Uplink Operations Teams. A Virtual Team is comprised of a group of people with the necessary mix of engineering and science expertise who come together for the purpose of building a specific uplink product. These people are drawn from throughout the Cassini Program and participate across a large geographical area (from Germany to the West coast of the USA), covering ten time zones. The participants will often split their time between participating in the Virtual Team and accomplishing their core responsibilities, requiring significant planning and time management. When the particular uplink product task is complete, the Virtual Team disbands and the members turn back to their home organization element for future work assignments. This time-sharing of employees is used on Cassini to build mission planning products, via the Mission Planning Virtual Team, and sequencing products and monitoring of the sequence execution, via the Sequence Virtual Team. This challenging, multitasking approach allows efficient use of personnel in a resource constrained environment.
\end{abstract}

KEYWORDS: Virtual Team, Distributed Operations, Uplink Operations, Sequencing, Mission Planning, Empowerment

\subsection{INTRODUCTION}

The Cassini Mission to Saturn is the last very large, flagship planetary orbiter mission currently budgeted by NASA. Launched on October 15, 1997, Cassini is on a 6.7 year cruise to Saturn, flying by Venus twice, the Earth, and finally Jupiter on a gravity- 
assist trajectory to gain enough energy to arrive at Saturn on July 1, 2004. At Saturn, Cassini's rich complement of 12 science instruments will spend four years taking observations of Saturn's rings, atmosphere, magnetosphere, icy satellites, and its largest moon, Titan. Additionally, Cassini is" carrying the European Space Agency's Huygens Probe mission, which has a payload of six instruments and will be deployed into the Titan atmosphere.

Cassini operations are conducted by the Cassini Program Office at the Jet Propulsion Laboratory (JPL) in Pasadena, California, USA. Huygens operations are conducted by the Huygens Probe Operations Centre (HPOC) at the European Space Operations Centre (ESOC) in Darmstadt, Germany. The Cassini Principal Investigators (PIs) and the Huygens Probe operators are located in six USA states (California, Arizona, Colorado, Texas, Iowa, and Maryland) and two European Countries (England and Germany) spanning a total of ten time zones. The flight system engineering is handled by personnel at JPL.

Key features of the Cassini mission are its cost-capped mission operations and development phase budgets, distributed operations, long cruise to Saturn and ambitious science objectives. A result of the cost caps is that Cassini has a smaller workforce than prior missions of similar scope. Hence, there are fewer Program organization elements than on past missions and more cross-trained personnel. Decision making authority is also kept to the lowest possible level. Cruise operations, although planned to be limited prelaunch, have become quite ambitious and include science data gathering at the Venus, Earth, and Jupiter flybys.

\subsection{VIRTUAL TEAM CONCEPT}

The Virtual Operations Team concept was born in an effort to reduce the cost of operations over the 11 year span of the mission, and to leverage the distributed nature of the Cassini Program. Instead of having personnel with similar skills in multiple Program elements, the Virtual Team concept calls for Program personnel to be shared or loaned to these uplink operations teams for the period of time that team is in existence (Figure 1). The Mission Planning Virtual Team (MPVT) is responsible for developing the Mission Plan and activity profile for each phase of the mission. The Sequence Virtual Team (SVT) is responsible for developing and executing integrated command sequences. Members of these Virtual Teams come from the Spacecraft Office (SCO), Real-time Operations Element (RTO), Uplink Operations Element (ULO), Mission Planning Element (MP) Instrument Operations Element (IO), Distributed Computing Services Element (DCS), Science Planning Element (SP), HPOC, and the PI Teams (Figure 2). Virtual Team members are trained in their home element discipline and go through a class to learn the Virtual Team processes and procedures.

A Virtual Team is formed to achieve a specific task and is in existence only for the duration of that task. Each Virtual Team member brings a specific expertise to the team. Membership to the Virtual Teams changes depending on the activities involved in the mission phase or sequence. For example, time periods with spacecraft maneuvers require the Virtual Teams to have membership from the Attitude and Articulation Control and Propulsion Team of the SCO. Time periods with instrument activities require the 
Virtual Team to have membership from the particular instrument team. When the particular uplink product task is complete, the Virtual Team disbands and the members revert to their home element for future work assignments. The next Virtual Team will typically have a different member composition based on the activities in their products.

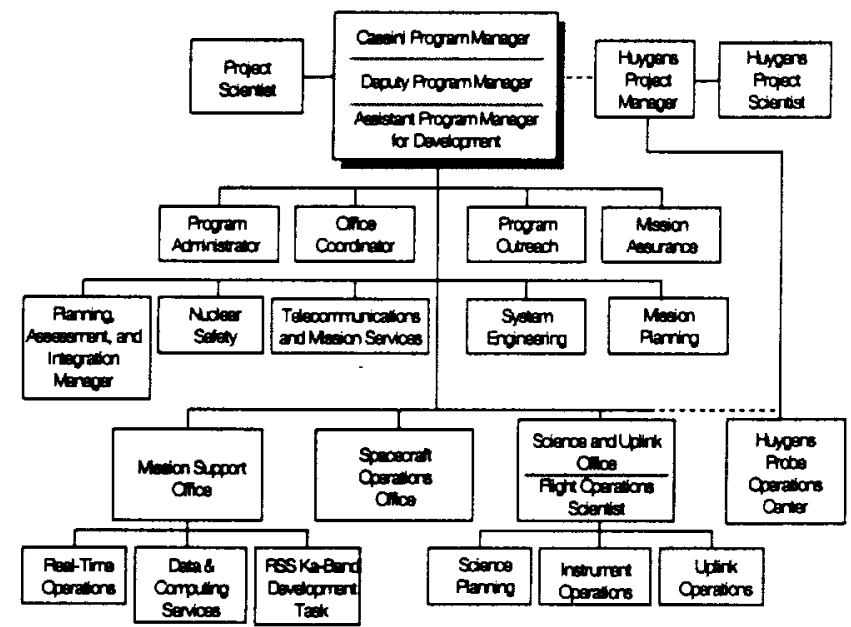

Figure 1: Cassini Program Organization

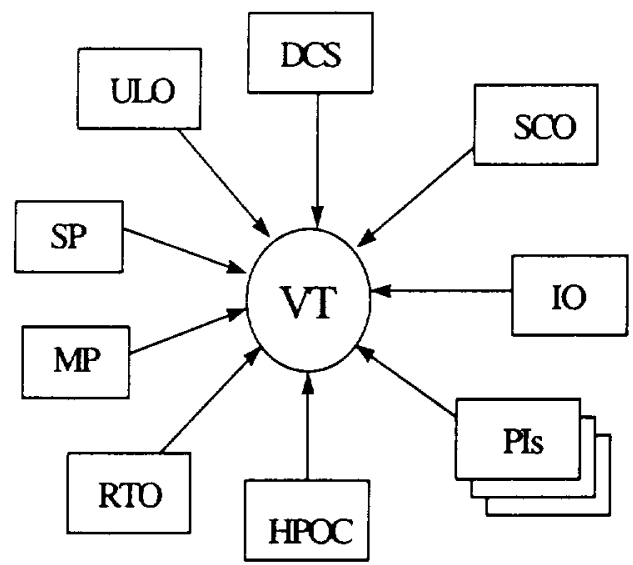

Figure 2: Virtual Team Composition

\subsection{VIRTUAL TEAM OPERATIONS}

The MPVT is lead by a member from the Mission Planning Element. The SVT is lead by a member from the Uplink Operations Element. The MP and ULO managers are responsible for negotiating with the other Program elements the personnel needed to support each Virtual Team. Once a Virtual Team is initiated, the team reports directly to the Program Manager and is responsible to him for product schedule and technical content. The Virtual Team lead is empowered to approve all products produced by the team, with the exception of waivers to a small set of critical spacecraft health and safety flight rules. Virtual Team members are empowered to represent their home element in the development of the Virtual Team products.

Virtual Teams communicate using a combination of email, teleconferenced meetings, and web pages and data bases available electronically. Since the team members come from many time zones, all meetings are held at 8 am Pacific Time, or earlier, so as not to be too late for the European sites. The Virtual Teams also employ well-defined processes and standardized meeting agendas to facilitate communications among team members and between successive Virtual Teams.

\subsection{MISSION PLANNING VIRTUAL TEAM}

The MPVT is responsible for updating the Mission Plan document by mission phase, generally a 6 to 12 month time period. The Mission Plan is distributed electronically to all members of the Program. The MPVT also produces a Phase Update Package (PUP) which contains a timeline for the phase, time-ordered listing of activities, 
information on consumable usage, contingency plans, trade study results, waivers, and change request

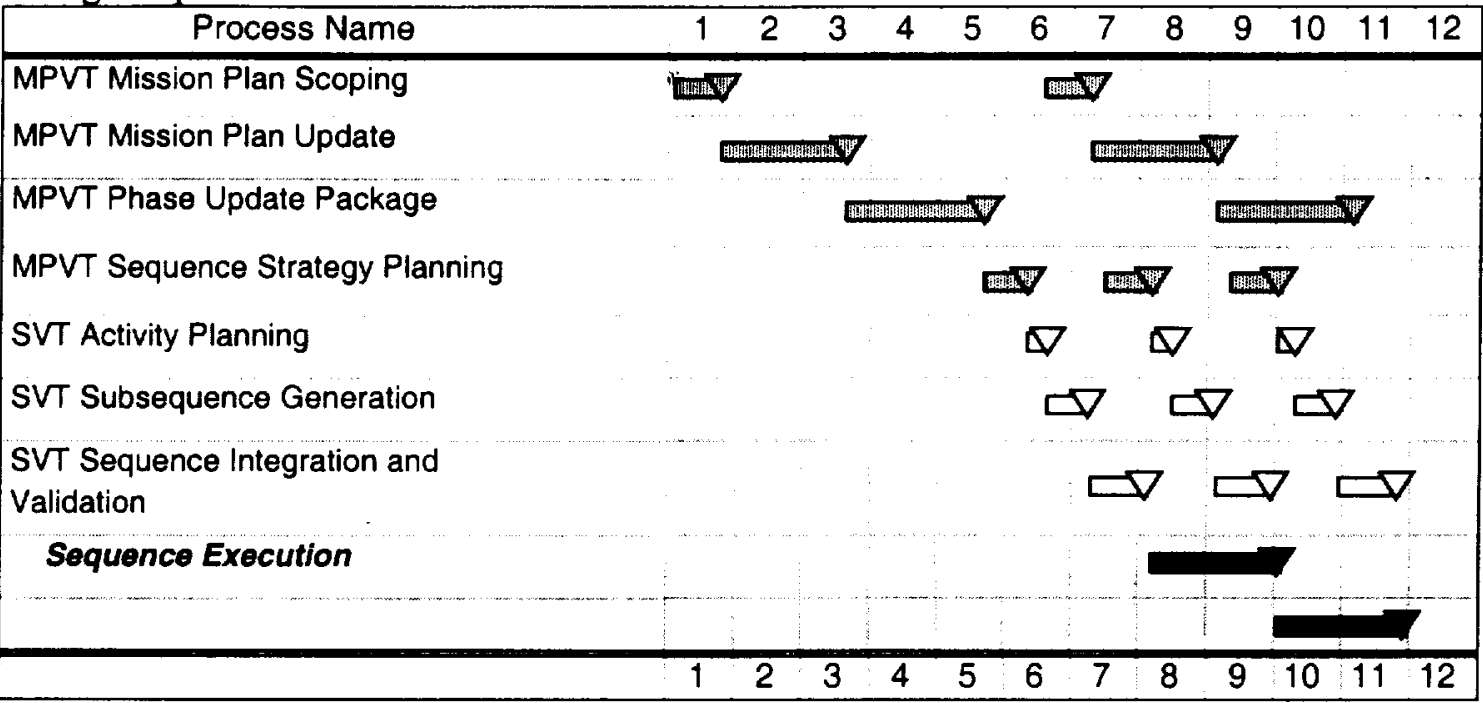

Figure 3: Example Cassini Uplink Schedule in Months

documentation. The PUP is made primarily for the SVT as a starting point from which to build the command sequences.

The MPVT process is broken into four parts. First is the Mission Plan scoping process, lasting three weeks. Here, requests for new, unplanned activities during the upcoming mission planning phase are input to the MPVT. These activities are generally science events, but can also include new spacecraft engineering requests. The MPVT reviews the requests for feasibility of implementation and then recommends to the Program Manager approval or disapproval of each request. Approved activities get included into the Mission. Plan update process, which is done over eight weeks. Following the Mission Plan update is the PUP generation, which takes nine weeks. During the PUP process the MPVT lays out a timeline of the activities in the phase and performs preliminary constraint checking. The final MPVT process is the Sequence Strategy Planning process (SSP) which occurs over the three weeks just prior to the start of the SVT process. During the SSP process, the MPVT updates the PUP for the upcoming sequence with the latest Deep Space Network (DSN) allocations and incorporates any changes to activities since the PUP was released. (Figure 3).

Since the MPVT products cover a 6 to 12 month time period, it contains a wide variety of spacecraft and instrument activities, and spans multiple command sequences (sequences are eight weeks in duration). Thus, the MPVT membership usually contains representatives from all the Program elements, including the relevant SVT leads from the ULO. The MPVT holds teleconferenced meetings once every two weeks. The MPVT lead is empowered to approve the Mission Plan update and the PUP.

\subsection{SEQUENCE VIRTUAL TEAM}


The SVT is responsible for both the generation and execution of command sequences. The SVT takes the PUP from the MPVT and produces constraint checked sequence files which are subsequently radiated to the spacecraft for execution. A typical sequence takes eight weeks to generate and eight weeks to execute. Thus, there are always two SVT's active at any time, one working on the current sequence execution and the other working on the generation of the next sequence.

The eight week sequence generation process is divided into three subphases: activity planning (AP), subsequence generation (SSG), and sequence integration and validation (SI\&V). During activity planning, the SVT lead schedules activities to the minute resolution, closes liens from the MPVT product, and distributes the sequence electronically for review by team members. The team members then generate and constraint check their individual command files in the subsequence generation phase. These subsequences are merged, integrated, system constraint checked, and validated by the SVT lead in the final SI\&V subphase (Figure 3).

The SVT membership will vary depending on the activities scheduled in the sequence. Typically, the SVT will be composed of members from SCO, RTO, ULO, IO, DCS, SP, HPOC, and some, but not all, PI teams. The SVT holds a kickoff teleconferenced meeting at the start of the AP process, and approval meetings at the end of each of the three subphases. The SVT Lead is responsible for product generation as well as for negotiating all resources to carry out a sequence, such as DSN coverage, and is empowered to approve command sequence radiation to the spacecraft. During sequence execution, the SVT is responsible for the generation and execution of real-time commands, and for reporting status on spacecraft activities to the Project management.

\subsection{COMPARISION OF CASSINI TO GALILEO AND VOYAGER PROJECTS}

The Cassini mission is the most ambitious and complicated robotic probe flown to date. It is comparable in size and mission scope to the Galileo mission to Jupiter and the Voyager missions to the outer planets. Yet the Cassini budget in real-year dollars is less than either of these two other missions. The Virtual Team concept is one reason for the cost savings as compared to Galileo and Voyager.

During the Galileo interplanetary cruise to Jupiter, the sequence generation process took 10 to 12 weeks, employing 10 people. For the Voyager Uranus to Neptune cruise, the generation process was 12 weeks and employed approximately 10 people. On Cassini, the sequence generation process takes eight weeks with the equivalent of six fulltime people supporting each SVT. All projects had the same two month cruise sequence execution time.

The Cassini MPVT has five full-time equivalent members supporting the uplink process. Both the Galileo and Voyager mission planning teams also had five full-time members. However, the Galileo and Voyager cruise uplink planning products were not to the same level of detail as that of the Cassini MPVT. The Cassini MPVT builds a timeline of activities to the day resolution for each phase, where as on Galileo and Voyager there are no cruise timelines, but rather simply lists of required activities and approximate timing. The duration of the mission planning process is also slightly shorter 
on Cassini, lasting a total of 23 weeks. The Galileo and Voyager mission phase update processes usually took six months (26 weeks).

One major reason for the shorter generation time is the empowerment of the Virtual Teams. Except for waivers in a few critical areas, no Element or Program Manager approvals are needed during the course of the sequence development process. The SVT lead and SVT members are closest to the details of the sequence, and thus have the best technical knowledge of the products. They are empowered by the Program to make decisions which on Galileo and Voyager were made by higher level managers. The MPVT is empowered to make mission planning decisions which are within the scope of the current Program budget and workforce resources.

The use of distributed operations and the sharing of Virtual Team personnel with their home element allows Cassini to have less workforce for uplink product generation than past missions. Another factor is the use of workstation based software tools to generate the sequences which allows more automation of processes and quicker generation of uplink products. For Cassini, there are no dedicated software operators, and thus there is a reduction in needed personnel. The same members of the SVT who design the observations also generate and validate the software and sequence products. This was not the case on either Voyager or Galileo, where there were multiple hand-offs between different teams during the generation process.

\subsection{VIRTUAL TEAMS EXPERIENCE TO DATE}

The Cassini spacecraft has been in flight for over 15 months and has been operating exceptionally well. The Virtual Team concept and distributed operations are also functioning very well. The use of email and teleconferencing for communications has been deemed a success by all parties involved. The sharing of personnel with their home organization has gone well, although specific agreements between the Virtual Team and their home element managers are necessary to insure proper member training and participation levels.

It is the authors' opinion that the SVT has been more successful in operating than the MPVT. Since the SVT starts with an agreed plan, its process has functioned more smoothly than the MPVT process. The SVT has generated 12 cruise sequences and over 600 real-time command files. There have been only two spacecraft safing incidents, and in both cases the SVT was able to restore the spacecraft to normal operations within 72 hours with no loss of planned activities.

\subsection{SUMMARY}

The Cassini Virtual Teams have proven themselves to be an effective and efficient mechanism for operating a spacecraft with distributed sites to reduce costs. As with any new system, improvements are always being made to the Virtual Team processes, but its basic premise of time-sharing employees and empowering team members has proven to be highly successful. The Virtual Team concept will continue to be used throughout the remaining 5.5 years of cruise, as well as during the four year orbital Tour of the Saturnian system. 


\section{ABBREVIATIONS:}

$\begin{array}{ll}\text { AP } & \text { Activity Planning } \\ \text { DCS } & \text { Distribute Computing Services Element } \\ \text { DSN } & \text { Deep Space Network } \\ \text { ESOC } & \text { European Space Operations Centre } \\ \text { HPOC } & \text { Huygens Probe Operations Centre } \\ \text { IO } & \text { Instrument Operations Element } \\ \text { JPL } & \text { Jet Propulsion Laboratory } \\ \text { MP } & \text { Mission Planning Element } \\ \text { MPVT } & \text { Mission Planning Virtual Team } \\ \text { NASA } & \text { National Aeronautic and Space Administration } \\ \text { PI } & \text { Principal Investigator } \\ \text { PUP } & \text { Phase Update Package } \\ \text { RSS } & \text { Radio Science Subsystem } \\ \text { RTO } & \text { Real-time Operations Element } \\ \text { SCO } & \text { Spacecraft Office } \\ \text { SI\&V } & \text { Sequence Integration and Validation } \\ \text { SP } & \text { Science Planning Element } \\ \text { SSG } & \text { Subsequence Generation } \\ \text { SSP } & \text { Sequence Strategy Planning } \\ \text { SVT } & \text { Sequence Virtual Team } \\ \text { ULO } & \text { Uplink Operations Element } \\ \text { USA } & \text { United States of America }\end{array}$

\section{REFERENCES}

1. Cassini Program, Uplink Operations Element, Cruise Mission Planning Operations Concept, PD 699-500-3-GS/D, 12 August 1997 (JPL internal document).

2. Cassini Program, Uplink Operations Element, Cruise Sequence Operations Concept, Revision C, PD 699-500-4-ULO/D, 15 June 1998 (JPL internal document).

3. "Distributed Operations for the Cassini/Huygens Mission", P. Lock and M. Sarrel, SpaceOps 98 Conference, Tokyo, 1-5 June, 1998, Paper ID: 1 b002

4. Cassini Mission Plan, Jet Propulsion Laboratory, California Institute of Technology, Revision I, PD 699-100, 18 December 1998 (JPL internal document).

5. Cassini Home Page (http://www.jpl.nasa.gov/cassini)

The research described in this paper was carried out by the Jet Propulsion Laboratory, California Institute of Technology, under a contract with the National Aeronautics and Space Administration. 